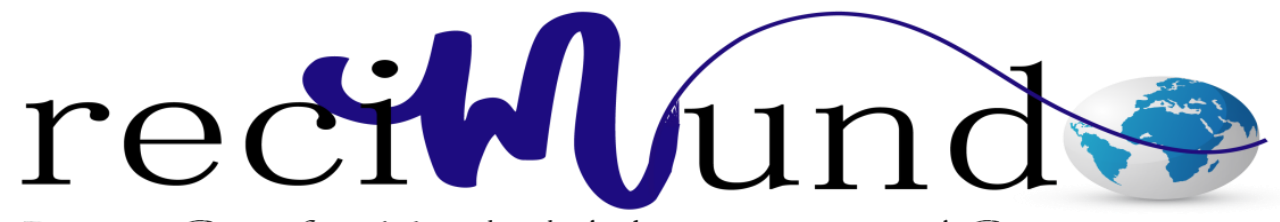

Revista Científica Mundo de la Investigación y el Conocimiento

Stefania Venegas Sánchez ${ }^{\text {a }}$ Diego Orellana Bueno ${ }^{\text {b; }}$ Pablo Pérez Jara ${ }^{\mathrm{c}}$

La realidad Ecuatoriana en la producción de café

Revista Científica Mundo de la Investigación y el Conocimiento. Vol. 2 núm.2, mayo, ISSN: 2588-073X, 2018, pp. 72-91

DOI: 10.26820/recimundo/2.(2).2018.72-91

Editorial Saberes del Conocimiento

Recibido: 05/12/2017

Aceptado: 20/03/2018
a. tifanies_27@hotmail.com
b. dvorellana77@gmail.com
c. perezpjp@gmail.com 


\section{La realidad Ecuatoriana en la producción de café}

Vol. 2, núm. 2., (2018)

Stefania Venegas Sánchez; Diego Orellana Bueno; Pablo Pérez Jara

\section{RESUMEN}

En la presente investigación, se ha hecho un abordaje sobre la producción cafetalera en Ecuador y los procesos que la integran. Ecuador es un país con tradición en el cultivo del café tanto así que es uno de los pocos países que produce dos tipos de café, el Arábico y Robusta, sin embargo la producción de café ha sufrido una vertiginosa caída desde los años 90 que no ha podido ser recuperada hasta la fecha. La metodología de investigación es de tipo documental y no experimental. De entre todas las conclusiones que se pueden mencionar es que la gran baja producción que ha experimentado el café está asociada a factores como: limitada capacidad organizativa de los productores, presencia de intermediarios locales, falta de conocimiento del germoplasma cultivado, una incipiente tecnificación, falta de acceso a crédito, la falta de infraestructura para acceder a las plantaciones de café, infraestructura para riego y condicione climáticas. Entre algunas de las recomendaciones es que hay que seguir capacitando a los productores de café, de igual manera hay que apoyarlos con financiamiento, semillas y fertilizantes.

Palabras claves: Producción, Cultivo, Productores, Café, Arábico. 


\title{
La realidad Ecuatoriana en la producción de café
}

Vol. 2, núm. 2., (2018)

Stefania Venegas Sánchez; Diego Orellana Bueno; Pablo Pérez Jara

\begin{abstract}
In the present investigation, an approach has been made about the coffee production in Ecuador and the processes that integrate it. Ecuador is a country with a tradition in the cultivation of coffee so much so that it is one of the few countries that produces two types of coffee, the Arabica and Robusta, however the coffee production has suffered a vertiginous fall since the 90s that has not could be recovered to date. The research methodology is documentary and not experimental. Among all the conclusions that can be mentioned is that the great low production that coffee has experienced is associated to factors such as: limited organizational capacity of the producers, presence of local intermediaries, lack of knowledge of the cultivated germplasm, an incipient technification, lack of access to credit, the lack of infrastructure to access coffee plantations, infrastructure for irrigation and climatic conditions. Among some of the recommendations is that we must continue to train coffee producers, in the same way we must support them with financing, seeds and fertilizers.
\end{abstract}

Keywords: Production, Cultivation, Producers, Coffee, Arabica. 


\section{La realidad Ecuatoriana en la producción de café}

Vol. 2, núm. 2., (2018)

Stefania Venegas Sánchez; Diego Orellana Bueno; Pablo Pérez Jara

\section{Introducción.}

El café tiene relevante importancia en los órdenes: económico, social, ambiental y salud humana. En lo económico, es una fuente de divisas para el país e ingresos para productores y otros actores de la cadena que en el 2015 representó ingresos por USD 145.354.370,31, según estadísticas del Ministerio de Agricultura, Ganadería, Acuacultura y Pesca del Ecuador. En lo social, la caficultura involucra a casi todas las etnias como kichwas, Shuaras y Tsáchilas, que se arraigan en un amplio tejido social (PROECUADOR, 2013, p.6). En lo ambiental, el café se cultiva básicamente en sistemas agroforestales y contribuye a la conservación de los recursos naturales y biodiversidad. En lo relacionado a la salud, Gotteland y De Pablo (2007) y Capel et al (2010) indican que el consumo de café muestra correlación inversa con el riesgo de diabetes tipo 2, daño hepático y enfermedades neurodegenerativas como el Parkinson (Guambi, Talledo, \& Ávila, 2016, pag. 15 y 16).

El café, en el Ecuador, es un cultivo de gran importancia económica, ya que cuenta con 199. 215 ha cultivadas, el $68 \%$ de esta área corresponde a la especie Coffea arábica y el $32 \%$ a Coffea canephora (PRO ECUADOR, 2013). El cultivo de café está distribuido en 23 de las 24 provincias del país, por lo tanto está relacionado con un amplio tejido social. C. arábica recibe el nombre de café arábigo y es considerado el de mejor calidad, su producción se concentra en las provincias de Manabí (especialmente en la localidad de Jipijapa), Loja y en las estribaciones de la Cordillera Occidental de los Andes. En cambio, C. canephora, llamado café robusta, se cultiva mayormente en la Amazonía, es decir en Sucumbíos y Orellana (Aspiazu \& Navarro, 2009) (Santistevan, Julca, Borjas, \& Tuesta, 2014, pag. 187) 


\section{La realidad Ecuatoriana en la producción de café}

Vol. 2, núm. 2., (2018)

Stefania Venegas Sánchez; Diego Orellana Bueno; Pablo Pérez Jara

Según la Organización Internacional del Café (ICO) por sus siglas en inglés, para el año 2015 Ecuador estaba dentro del listado de los principales productores de café en el mundo, para ese año, el país se ubicada en la posición número 19 de un total de 20 países, con una participación del 0,49\% de la producción mundial, con un total de 42.000 kilos, muy por debajo de países con tradición cafetalera en Latinoamérica como Brasil (puesto $\mathrm{N}^{\circ}$ 1) y Colombia (puesto $\mathrm{N}^{\circ}$ 3).

El cultivo de café en Ecuador posee tanto valor económico, como social y ecológico. La importancia social y económica se basa en la generación de empleo para 105.000 familias de productores; así como para 700.000 familias adicionales vinculadas a los procesos de comercialización, industrialización, transporte y exportación. En el orden ecológico, la importancia del café radica en la amplia adaptabilidad de los cafetales a los distintos agro ecosistemas de las cuatro regiones del país: Costa, Sierra, Amazonía e Islas Galápagos (COFENAC, 2011) (Alarcó, 2011, pag. 1).

La actividad agroproductiva del café presenta varios factores que afectan la productividad y la calidad del grano, por repercusiones como la inestabilidad de los precios en el mercado mundial, fenómenos naturales como «El Niño», reducción del área cultivada y edad avanzada de los cafetales; todo lo cual, incide negativamente en la economía de los productores (Vaca, Suarez, \& Velázquez, 2016, pag. 121).

El presente trabajo de investigación busca hacer un abordaje sobre la produccion de café en nuestro pais, tendientes a identificar cuales son sus potencialidades, y que estrategias se deben emplear para potenciar la produccion de este rubro en Ecuador 


\section{La realidad Ecuatoriana en la producción de café}

Vol. 2, núm. 2., (2018)

Stefania Venegas Sánchez; Diego Orellana Bueno; Pablo Pérez Jara

\section{Materiales y Metodos}

La presente investigación es de tipo documental y no experimental. Documental, ya que busca las opiniones de otros autores sobre la materia de estudio, en base a la investigación por medios electronicos, que luego seran utilizados como referencias bibliograficas. Es no experimetal, ya que no se esta haciendo un estudio de campo, las opiniones aquí expresadas son de los autores mencioandos y en ningun momento se han manipulado sus aseveraciones, lo que se busca mas bien es hacer analisis de los resultados presentados para de esta manera prsentar las conclusiones y recomedaciones necesarias.

\section{Resultados}

Imagen $N^{\circ}$ 1: Características óptimas para el desarrollo de un cafetal

\begin{tabular}{|c|c|}
\hline \multicolumn{2}{|c|}{ CARACTERÍSTICAS ÓPTIMAS PARA EL DESARROLLO DE UN CAFETAL } \\
\hline ALTITUD & $\begin{array}{l}\text { La altitud óptima se localiza entre los } 1.200 \text { y } 1.700 \mathrm{msnm} \text {, aunque en } \\
\text { Ecuador se han legado a establecer desde los } 300 \mathrm{msnm} \text { y por encima de los } \\
1.700 \mathrm{msnm} \text { con buenos rendimientos. }\end{array}$ \\
\hline TEMPERATURA & Entre $\operatorname{los} 15$ y $24^{\circ} \mathrm{C}$ \\
\hline PRECIPITACIONES & $\begin{array}{l}\text { El rango de precipitaciones óptimas para el cultivo del café puede variar de } \\
1.000 \mathrm{a} 3.000 \mathrm{~mm} \text {. }\end{array}$ \\
\hline $\begin{array}{l}\text { HUMEDAD } \\
\text { RELATIVA }\end{array}$ & En torno al $70-85 \%$ \\
\hline VIENTO & $\begin{array}{l}\text { El umbral para no producir daños físicos ni fisiológicos al cafeto es de } 20 \text { a } \\
30 \mathrm{~km} / \mathrm{hora} \text {. }\end{array}$ \\
\hline TOPOGRAFÍA & $\begin{array}{l}\text { Se adapta con facilidad a condiciones topográficas desfavorables, aunque } \\
\text { los terrenos ligeramente ondulados y planos son mejores para este cultivo. }\end{array}$ \\
\hline \multirow[b]{2}{*}{$\begin{array}{l}\text { CARACTERÍSTICAS } \\
\text { FÍSICO-QUIMIICAS }\end{array}$} & $\begin{array}{l}\text { Propiedades Físicas: La textura adecuada para el cafetal es media o limosa, } \\
\text { con estructura granular. La profundidad efectiva debe ser mayor de } 50 \mathrm{~cm} \text {. }\end{array}$ \\
\hline & $\begin{array}{l}\text { Propiedades químicas: El rango de } \mathrm{pH} \text { óptimo se encuentra entre } 5,5 \text { y } 6,5 \text {. } \\
\text { El porcentaje de materia orgánica se debe encontrar en } 2-5 \% \text {, y el de } \\
\text { nitrógeno superior al } 3 \% \text { para el adecuado desarrollo del café. Las } \\
\text { condiciones óptimas en cuanto a macronutrientes para este cultivo son de } \\
0,2-0,7 \text { (meq/100gr) en el caso del potasio y } 6-14(\mathrm{ppm}) \text { en el caso del } \\
\text { fósforo. }\end{array}$ \\
\hline
\end{tabular}

Fuente: (Alarcó, 2011, pag. 8) 


\section{La realidad Ecuatoriana en la producción de café}

Vol. 2, núm. 2., (2018)

Stefania Venegas Sánchez; Diego Orellana Bueno; Pablo Pérez Jara

Existen cinco categorías para clasificar a los sistemas cafetales en función de su diversidad y complejidad (Moguel y Toledo, 1999):

a) Sistema tradicional o rústico: donde los cafetos se insertan dentro de un bosque ya existente.

b) Sistema de policultivo tradicional: cafetal cultivado bajo sombra menos densa constituida por especies forestales de valor medicinal.

c) Sistema de policultivo comercial: los cafetos se encuentran bajo sombra de bananeros y frutales.

d) Sistema bajo sombra especializada y fuertemente controlada: cafetal bajo sombra de una o dos especies forestales, normalmente leguminosas.

e) Monocultivo: cultivo de café expuesto totalmente a radiación solar (Alarcó, 2011, pag. 9)

Tabla $\mathbf{N}^{\circ}$ 1: Proceso Productivo del Café

\begin{tabular}{|c|l|}
\hline Procesos & \multicolumn{1}{c|}{ Descripción } \\
\hline Germinador & $\begin{array}{l}\text { La semilla debe estar disponible } 8 \text { meses antes del trasplante definitivo en campo, de } \\
\text { este período, dos meses corresponden a la etapa de germinador y seis meses al } \\
\text { almácigo. En las etapas del proceso productivo de germinador y almácigo se necesitan } \\
\text { insumos que se pueden adquirir en la zona, como guaduas o estacones de madera } \\
\text { redonda, arena lavada de río, tierra, pulpa descompuesta o materia orgánica, bolsas } \\
\text { plásticas, materiales para disponer sombra en viveros, plaguicidas de baja toxicidad, } \\
\text { solo si se requieren. }\end{array}$ \\
\hline
\end{tabular}




\begin{tabular}{|c|c|}
\hline Siembra & $\begin{array}{l}\text { Se siembra la semilla, la germinación ocurre después de } 45-50 \text { días, y se le llaman } \\
\text { fósforos, } 15 \text { días después empiezan a salir las primeras hojas, el nombre que reciben es } \\
\text { chapolas, luego se trasplantan en bolsas negras al almacigo, que consiste en una } \\
\text { estructura que protege a los futuros árboles del sol y la lluvia. Entre 150-180 días, los } \\
\text { árboles se trasplantan al campo. }\end{array}$ \\
\hline Almacigo & $\begin{array}{l}\text { Su finalidad es el desarrollo adecuado y la selección de las plántulas para el } \\
\text { establecimiento definitivo del cultivo. }\end{array}$ \\
\hline Fertilización & $\begin{array}{l}\text { Comprende las etapas de instalación, establecimiento, crecimiento y producción. Esta } \\
\text { práctica se complementa con adiciones sucesivas de materia orgánica, principalmente } \\
\text { por el manejo de subproductos del cultivo. Control de plagas. Dentro de los controles } \\
\text { utilizados para el manejo de plagas en café se encuentran el biológico, el cultural, el } \\
\text { legal, el mecánico, el natural y el químico. }\end{array}$ \\
\hline $\begin{array}{c}\text { Manejo de } \\
\text { Enfermedades }\end{array}$ & $\begin{array}{l}\text { El manejo integrado de enfermedades requiere la ejecución oportuna de las diferentes } \\
\text { prácticas de cultivo. Esto involucra adecuada nutrición, desyerbas a tiempo y un } \\
\text { concepto interiorizado en el caficultor de producir un café libre de enfermedades y de } \\
\text { calidad, sin que se afecten las relaciones con el medio ambiente. }\end{array}$ \\
\hline Floración & $\begin{array}{l}\text { Entre los primeros } 540-600 \text { días se da la primera floración, y 240-270 días más tarde se } \\
\text { da el primer fruto maduro }\end{array}$ \\
\hline Recolección & $\begin{array}{l}\text { La recolección se hace manualmente, el café esta plantado principalmente en laderas. } \\
\text { Con el propósito de mejorar la calidad, los recolectores solo recogen los granos } \\
\text { maduros. }\end{array}$ \\
\hline Cosecha & $\begin{array}{l}\text { Se deben recoger, en su totalidad, sólo los frutos maduros. Esto se traduce en las } \\
\text { siguientes ventajas: aumento de ingresos por venta de mayor cantidad de café (mejor } \\
\text { conversión de café cereza: café pergamino seco). }\end{array}$ \\
\hline Despulpar & $\begin{array}{l}\text { Los productores usan la despulpadora para quitar la cereza del grano, el agua es el } \\
\text { principal factor usado en esta etapa. Esta máquina clasifica los granos por peso y } \\
\text { tamaño. }\end{array}$ \\
\hline Fermentación & $\begin{array}{l}\text { Este proceso que usualmente tiene un tiempo entre } 18-24 \text { horas, se realiza dentro de } \\
\text { tanques de agua. Con la fermentación el mucílago o capa viscosa se descompone. }\end{array}$ \\
\hline
\end{tabular}




\section{La realidad Ecuatoriana en la producción de café}

Vol. 2, núm. 2., (2018)

Stefania Venegas Sánchez; Diego Orellana Bueno; Pablo Pérez Jara

\begin{tabular}{|c|l|}
\hline Lavado & $\begin{array}{l}\text { Se realiza en los mismos tanques o en canales ubicados en el BENEFICIO, con el } \\
\text { lavado se elimina el mucílago. }\end{array}$ \\
\hline $\begin{array}{c}\text { Remoción del } \\
\text { mucílago }\end{array}$ & $\begin{array}{l}\text { Tiene como fin hacer que el mucílago que cubre el pergamino se descomponga y una } \\
\text { vez fermentado se disuelva en agua, eliminándose por medio del lavado. Su duración } \\
\text { oscila entre } 18 \text { y } 30 \text { horas, según el clima }\end{array}$ \\
\hline Secado & $\begin{array}{l}\text { Este proceso se hace al sol. En algunas fincas se usa hornos que trabajan con energía, } \\
\text { carbón o madera. La humedad permitida para realizar la compra es de 12\% }\end{array}$ \\
\hline Trilla & $\begin{array}{l}\text { En este paso se elimina el pergamino, y dejando el grano verde listo para ser } \\
\text { clasificado por máquinas seleccionadoras que lo hacen por tamaño y peso. }\end{array}$ \\
\hline
\end{tabular}

Fuente: (Pozo, 2014, pag. 23,24 y 25)

Tabla $\mathbf{N}^{\circ}$ 2: Ecuador: superficie, producción y rendimiento del café

\begin{tabular}{|c|c|c|c|c|c|c|c|}
\hline Años & $\begin{array}{c}\text { Superficie } \\
\text { Cosechada } \\
\text { (ha) }\end{array}$ & $\begin{array}{l}\text { Producción } \\
\text { (TM) }\end{array}$ & $\begin{array}{c}\text { Rendimiento } \\
\text { (TM/ha) }\end{array}$ & $\begin{array}{c}\text { Producción en } \\
\text { quintales }\end{array}$ & $\begin{array}{c}\text { Rendimien- to } \\
\text { en quintales/ha }\end{array}$ & $\begin{array}{l}\text { Rendimien- to } \\
\text { en sacos } \\
60 \mathrm{Kg} / \mathrm{ha}\end{array}$ & \begin{tabular}{|c} 
Producción \\
Anual Total en \\
sacos \\
$60 \mathrm{Kg}$
\end{tabular} \\
\hline 1990 & 406.880 & 108.960 & 0,27 & 2.179 .200 & 5,28 & 4,0 & 1.648 .411 \\
\hline 1991 & 403.870 & 124.850 & 0,31 & 2.497 .000 & 6,18 & 4,7 & 1.888 .805 \\
\hline 1992 & 400.460 & 118.040 & 0,29 & 2.360 .800 & 5,89 & 4,4 & 1.785 .779 \\
\hline 1993 & 396.770 & 137.004 & 0,35 & 2.740 .080 & 6,90 & 5,2 & 2.072 .663 \\
\hline 1994 & 399.880 & 186.797 & 0,47 & 3.735 .940 & 9,34 & 7,0 & 2.826 .976 \\
\hline 1995 & 384.010 & 148.205 & 0,39 & 2.964 .100 & 7,72 & 5,8 & 2.242 .118 \\
\hline 1996 & 397.283 & 190.720 & 0,48 & 3.814 .400 & 9,60 & 7,2 & 2.885 .626 \\
\hline 1997 & 348.119 & 87.350 & 0,25 & 1.747 .000 & 5,02 & 3,8 & 1.321 .483 \\
\hline 1998 & 389.782 & 48.190 & 0,12 & 963.800 & 2,47 & 1,8 & 729.047 \\
\hline 1999 & 374.633 & 132.939 & 0,35 & 2.658 .780 & 7,10 & 3,2 & 1.210 .287 \\
\hline 2000 & 350.000 & 76.102 & 0,22 & 1.522 .040 & 4,35 & 3,2 & 1.149 .773 \\
\hline 2001 & 270.110 & $* 77080$ & 0,29 & 1.541 .600 & 5,70 & 3,93 & 1.061 .573 \\
\hline 2002 & 231.918 & 58.557 & 0,25 & 1.171 .140 & 5,05 & 3,82 & 885.885 \\
\hline
\end{tabular}

* Producción estimada según MAG.

FUENTE: Superficie Cosechada SICA-MAG www.sica.gov.ec.

Producción en TM hasta el 2000. Boletín Anuario N 23 del Banco Central del Ecuador, excepto años 1990-1992 proporcionados por COFENAC, así como la producción en sacos de 60kg de los años 1990 a 1992.

ELABORACIÓN: Jaime Ortega Bardellini

Fuente: (Ortega, 2003, pag 29) 


\section{La realidad Ecuatoriana en la producción de café}

Vol. 2, núm. 2., (2018)

Stefania Venegas Sánchez; Diego Orellana Bueno; Pablo Pérez Jara

Como se puede observar en el siguiente cuadro se presenta la produccion en un periodo de 12 años de café en Ecuador, se destacan dos momentos, el primero desde 1990 hasta 1996 con una aumento sostenido de la produccion de un $75,05 \%$ con una tasa de crecimiento de esos 6 años de un 9,78\%. El segundo momento es a partir del 1997 hasta el año 2002 donde empieza una caida de la produccion de un $32,96 \%$. El mismo comportamiento se observa en la cantidad de superficies cosechadas en hectareas en esos 12 años disminuyeron las hectareas en un 43\%, con una tasa de decrecimiento de un 5,04\%.

Ortega (2003), indica las causas posibles de ese decrecimiento sostenido, cuando afirma: "Esta disminución en el área cosechada se debe a que para cada una de las actividades culturales que comprende el proceso productivo del café, se han presentado dificultades que han impedido a los precios locales cubrir los costos. Otra explicación es la caída de precios a nivel internacional, por la cual los caficultores han optado por sustituir sus cafetales por otro tipo de cultivos que les resulten más rentables. (p. 29).

Tabla $\mathbf{N}^{\circ}$ 3: Ecuador: superficie cafetalera y proyección de cosecha al año 2002

\begin{tabular}{|c|c|c|c|c|c|c|c|c|c|}
\hline \multirow[t]{4}{*}{ Provincias } & \multicolumn{4}{|c|}{ ARÁBIGO } & \multicolumn{2}{|c|}{ ROBUSTA } & \multirow{2}{*}{\multicolumn{2}{|c|}{ TOTAL }} & \multirow[b]{3}{*}{$\begin{array}{l}\text { Produc- } \\
\text { ción }\end{array}$} \\
\hline & $\begin{array}{c}\text { Superfi- } \\
\text { cie }\end{array}$ & & \begin{tabular}{|c|}
$\begin{array}{c}\text { Produc- } \\
\text { ción }\end{array}$ \\
\end{tabular} & & Superficie & Producción & & & \\
\hline & $\begin{array}{c}\text { Hectá- } \\
\text { reas }\end{array}$ & \begin{tabular}{|l|} 
Lavado \\
\end{tabular} & Natural & Subtotal & Hectáreas & Pilado & Superficie & $\begin{array}{l}\text { Produc- } \\
\text { ción }\end{array}$ & \\
\hline & & qq & $\mathbf{q q}$ & qq & & qq & Hectáreas & $q q$ & $\%$ \\
\hline Esmeraldas & 5.000 & 15.000 & 5.000 & 20.000 & 4.800 & 33.600 & 9.800 & 53.600 & $5 \%$ \\
\hline Manabí & 70.000 & 110.000 & 120.000 & 230.000 & 300 & 1.800 & 70.300 & 231.800 & $20 \%$ \\
\hline Guayas & 15.000 & 25.000 & 35.000 & 60.000 & 1.000 & 5.000 & 16.000 & 65.000 & $6 \%$ \\
\hline Los Ríos & 6.500 & 4.000 & 25.000 & 29.000 & 5.000 & 39.000 & 11.500 & 68.000 & $6 \%$ \\
\hline El Oro & 10.000 & 11.000 & 40.000 & 51.000 & 0 & 0 & 10.000 & 51.000 & $4 \%$ \\
\hline Carchi & 50 & 100 & 100 & 200 & 0 & 0 & 50 & 200 & $0 \%$ \\
\hline Imbabura & 450 & 1.300 & 1.200 & 2.500 & 1.500 & 10.000 & 1.950 & 12.500 & $1 \%$ \\
\hline Pichincha & 2.000 & 2.800 & 6.500 & 9.300 & 8.000 & 50.000 & 10.000 & 59.300 & $5 \%$ \\
\hline Cotopaxi & 1.500 & 1.000 & 7.000 & 8.000 & 3.700 & 27.000 & 5.200 & 35.000 & $3 \%$ \\
\hline Tungurahua & 0 & 0 & 0 & 0 & 0 & 0 & 0 & 0 & $0 \%$ \\
\hline Bolívar & 4.000 & 4.500 & 18.000 & 22.500 & 2.700 & 19.000 & 6.700 & 41.500 & $4 \%$ \\
\hline
\end{tabular}




\section{La realidad Ecuatoriana en la producción de café}

Vol. 2, núm. 2., (2018)

Stefania Venegas Sánchez; Diego Orellana Bueno; Pablo Pérez Jara

\begin{tabular}{|c|c|c|c|c|c|c|c|c|c|}
\hline Chimborazo & 500 & 1.300 & 1.500 & 2.800 & 0 & 0 & 500 & 2.800 & $0 \%$ \\
\hline Cañar & 700 & 1.000 & 2.200 & 3.200 & 150 & 600 & 850 & 3.800 & $0 \%$ \\
\hline Azuay & 400 & 700 & 1.200 & 1.900 & 20 & 90 & 420 & 1.990 & $0 \%$ \\
\hline Loja & 31.200 & 13.500 & 172.000 & 185.500 & 0 & 0 & 31.200 & 185.500 & $16 \%$ \\
\hline Sucumbios & 60 & 0 & 300 & 300 & 21.000 & 126.000 & 21.060 & 126.300 & $11 \%$ \\
\hline Orellana & 38 & 0 & 190 & 190 & 19.500 & 117.000 & 19.538 & 117.190 & $10 \%$ \\
\hline Napo & 120 & 0 & 600 & 600 & 11.250 & 67.200 & 11.370 & 67.800 & $6 \%$ \\
\hline Pastaza & 150 & 0 & 750 & 750 & 400 & 2.600 & 550 & 3.350 & $0 \%$ \\
\hline $\begin{array}{l}\text { Morona } \\
\text { Santiago }\end{array}$ & 90 & 80 & 360 & 440 & 600 & 3.600 & 690 & 4.040 & $0 \%$ \\
\hline Zamora & 3.500 & 28.000 & 9.000 & 37.000 & 40 & 270 & 3.800 & 37.270 & $3 \%$ \\
\hline Galápagos & 700 & 1.200 & 2.000 & 3.200 & 0 & 0 & 700 & 3.200 & $0 \%$ \\
\hline $\begin{array}{l}\text { TOTAL } \\
\text { QUINTALES }\end{array}$ & 151.958 & 220.480 & 447.900 & 668.380 & 79.960 & 502.760 & 231.918 & 1.171.140 & $100 \%$ \\
\hline $\begin{array}{l}\text { Total Sacos } \\
60 \mathrm{Kg}\end{array}$ & & 166.778 & 338.805 & 505.582 & & 380.303 & & 885.885 & \\
\hline
\end{tabular}

FUENTE: Proyecto MAG SICA-Ecuador www.sica.gov.ec.

NOTA: Hasta la actualidad SICA MAG mantiene el título como "Potencial de Producción".

Fuente: (Ortega, 2003, pag. 35)

Se observa para el momento del trabajo realizado por Ortega (2003), que Manabi y Loja son las provincias que mas hectareas tienen para la produccion de café en Ecuador. Manabi con una concetracion del $46,06 \%$ y Loja con un $20,53 \%$ es decir, ambas provincias poseian $66,59 \%$ del total de hectareas con capacidad productiva.

\section{Situación producción de café hasta hoy}

Tabla $\mathbf{N}^{\circ}$ 4: Producción, hectáreas plantadas y cosechadas de Café 2004 - 2013 por Regiones

\begin{tabular}{|c|c|c|c|c|c|c|c|c|c|c|}
\hline \multicolumn{10}{|c|}{ PRODUCCION EN TN DE CAFÉ 2003 - 2013 POR REGIONES } \\
\hline Descripción & $\mathbf{2 0 0 4}$ & $\mathbf{2 0 0 5}$ & $\mathbf{2 0 0 6}$ & $\mathbf{2 0 0 7}$ & $\mathbf{2 0 0 8}$ & $\mathbf{2 0 0 9}$ & $\mathbf{2 0 1 0}$ & $\mathbf{2 0 1 1}$ & $\mathbf{2 0 1 2}$ & $\mathbf{2 0 1 3}$ \\
\hline Región Costa & 307.073 & 304.623 & 278.490 & 265.425 & 251.056 & 254.420 & 246.296 & 155.525 & 127.422 & 56.123 \\
\hline Región Oriental & 13.412 & 17.953 & 11.797 & 17.335 & 11.447 & 11.080 & 10.776 & 9.353 & 3.836 & 2.211 \\
\hline Región Sierra & 12.583 & 18.947 & 15.796 & 16.445 & 11.585 & 12.259 & 12.514 & 10.240 & 5.252 & 2.178 \\
\hline Total & $\mathbf{3 3 5 . 0 7 3}$ & $\mathbf{3 4 3 . 5 2 8}$ & $\mathbf{3 0 8 . 0 9 0}$ & $\mathbf{3 0 1 . 2 1 2}$ & $\mathbf{2 7 6 . 0 9 6}$ & $\mathbf{2 7 9 . 7 6 9}$ & $\mathbf{2 7 1 . 5 9 6}$ & $\mathbf{1 7 7 . 1 2 9}$ & $\mathbf{1 3 8 . 5 2 2}$ & $\mathbf{6 2 . 5 2 5}$ \\
\hline & HECTAREAS PLANTADAS DE CAFÉ $\mathbf{2 0 0 3}$ & $\mathbf{2 0 1 3}$ POR REGIONES & & \\
\hline Descripción & $\mathbf{2 0 0 4}$ & $\mathbf{2 0 0 5}$ & $\mathbf{2 0 0 6}$ & $\mathbf{2 0 0 7}$ & $\mathbf{2 0 0 8}$ & $\mathbf{2 0 0 9}$ & $\mathbf{2 0 1 0}$ & $\mathbf{2 0 1 1}$ & $\mathbf{2 0 1 2}$ & $\mathbf{2 0 1 3}$ \\
\hline Región Costa & 307.073 & 304.623 & 278.490 & 265.425 & 251.056 & 254.420 & 246.296 & 155.525 & 127.422 & 56.123 \\
\hline Región Oriental & 91.342 & 95.148 & 78.289 & 84.562 & 64.784 & 64.342 & 60.451 & 35.480 & 37.568 & 16.011 \\
\hline
\end{tabular}




\section{La realidad Ecuatoriana en la producción de café}

Vol. 2, núm. 2., (2018)

Stefania Venegas Sánchez; Diego Orellana Bueno; Pablo Pérez Jara

\begin{tabular}{|c|c|c|c|c|c|c|c|c|c|c|}
\hline Región Sierra & 93.438 & 92.995 & 77.371 & 74.573 & 66.539 & 78.261 & 75.413 & 54.707 & 61.028 & 25.550 \\
\hline Total & $\mathbf{4 9 3 . 8 5 7}$ & $\mathbf{4 9 4 . 7 7 0}$ & $\mathbf{4 3 6 . 1 5 7}$ & $\mathbf{4 2 6 . 5 6 7}$ & $\mathbf{3 8 4 . 3 8 7}$ & $\mathbf{3 9 9 . 0 3 2}$ & $\mathbf{3 8 4 . 1 7 0}$ & $\mathbf{2 4 7 . 7 2 3}$ & $\mathbf{2 2 8 . 0 3 0}$ & $\mathbf{9 9 . 6 9 7}$ \\
\hline \multicolumn{7}{|c|}{ HECTAREAS COSECHADAS DE CAFÉ 2003 - 2013 POR REGIONES } \\
\hline Descripción & $\mathbf{2 0 0 4}$ & $\mathbf{2 0 0 5}$ & $\mathbf{2 0 0 6}$ & $\mathbf{2 0 0 7}$ & $\mathbf{2 0 0 8}$ & $\mathbf{2 0 0 9}$ & $\mathbf{2 0 1 0}$ & $\mathbf{2 0 1 1}$ & $\mathbf{2 0 1 2}$ & $\mathbf{2 0 1 3}$ \\
\hline Región Costa & 274.139 & 252.476 & 222.811 & 215.338 & 223.537 & 219.950 & 184.364 & 117.070 & 86.730 & 33.721 \\
\hline Región Oriental & 73.230 & 73.143 & 65.724 & 69.972 & 50.287 & 50.755 & 46.593 & 28.889 & 29.602 & 11.825 \\
\hline Región Sierra & 85.190 & 85.469 & 72.816 & 70.300 & 63.135 & 73.141 & 58.906 & 50.736 & 41.056 & 16.081 \\
\hline Total & $\mathbf{4 3 4 . 5 6 3}$ & $\mathbf{4 1 3 . 0 9 3}$ & $\mathbf{3 6 3 . 3 5 7}$ & $\mathbf{3 5 7 . 6 1 6}$ & $\mathbf{3 3 8 . 9 6 6}$ & $\mathbf{3 4 5 . 8 5 5}$ & $\mathbf{2 9 1 . 8 7 3}$ & $\mathbf{1 9 8 . 7 0 6}$ & $\mathbf{1 5 9 . 4 0 0}$ & $\mathbf{6 3 . 6 4 0}$ \\
\hline
\end{tabular}

Fuente: (Sistema Nacional de Informacion)

En la Tabla $\mathrm{N}^{\circ} 4$ se puede observar que la región costera es la que más hectáreas productivas de café posee, al igual que la plantación y cosecha. Se observa de igual manera el descenso continuado y vertiginoso de la cantidad de hectáreas disponibles para producir café, desde 1996 que ha sido el año con mayor cantidad de hectáreas disponibles hasta el año 2013 ha habido una disminución de un $\mathbf{2 . 7 9 4 \%}$

Tabla $\mathbf{N}^{\circ}$ 5: Hectáreas por provincias disponibles hasta el año 2013

\begin{tabular}{|c|c|c|c|c|}
\hline \multicolumn{7}{|c|}{ Hectáreas por Provincias Disponibles hasta el año 2013 } \\
\hline & $\begin{array}{c}\text { Hectáreas } \\
\text { Plantadas }\end{array}$ & $\begin{array}{c}\text { Hectáreas } \\
\text { Cosechadas }\end{array}$ & $\mathbf{\%}$ & $\begin{array}{c}\text { Producción } \\
\text { (TN) }\end{array}$ \\
\hline Provincias & 43,06 & 43,06 & $\mathbf{1 0 0 , 0 0}$ & 6,66 \\
\hline Bolívar & $3.129,42$ & $2.823,82$ & $\mathbf{9 0 , 2 3}$ & 448,49 \\
\hline Cañar & 0,00 & 0,00 & $\mathbf{0 , 0 0}$ & 0,00 \\
\hline Carchi & 186,91 & 100,41 & $\mathbf{5 3 , 7 2}$ & 8,60 \\
\hline Chimborazo & 21,27 & 8,44 & $\mathbf{3 9 , 6 8}$ & 2,22 \\
\hline Cotopaxi & $1.117,15$ & $1.094,31$ & $\mathbf{9 7 , 9 6}$ & 252,00 \\
\hline El Oro & $5.667,71$ & $2.021,24$ & $\mathbf{3 5 , 6 6}$ & 204,16 \\
\hline Esmeralda & 37,16 & 20,43 & $\mathbf{5 4 , 9 8}$ & 3,90 \\
\hline Guayas & $1.799,15$ & $1.069,47$ & $\mathbf{5 9 , 4 4}$ & 85,21 \\
\hline Imbabura & 11,42 & 8,80 & $\mathbf{7 7 , 0 6}$ & 2,21 \\
\hline Loja & $17.910,59$ & $9.719,09$ & $\mathbf{5 4 , 2 6}$ & $1.154,21$ \\
\hline Los Ríos & $3.609,64$ & $2.917,03$ & $\mathbf{8 0 , 8 1}$ & 161,61 \\
\hline Manabí & $\mathbf{4 4 . 6 0 7 , 7 6}$ & $\mathbf{2 7 . 6 0 7 , 3 7}$ & $\mathbf{6 1 , 8 9}$ & $\mathbf{2 . 9 1 0 , 6 6}$ \\
\hline Morona Santiago & 0,00 & 0,00 & $\mathbf{0 , 0 0}$ & 0,00 \\
\hline Napo & $2.658,00$ & $1.562,00$ & $\mathbf{5 8 , 7 7}$ & 150,00 \\
\hline
\end{tabular}




\section{La realidad Ecuatoriana en la producción de café}

Vol. 2, núm. 2., (2018)

Stefania Venegas Sánchez; Diego Orellana Bueno; Pablo Pérez Jara

\begin{tabular}{|c|c|c|c|c|}
\hline Orellana & $\mathbf{1 4 . 3 2 4 , 0 0}$ & $\mathbf{1 1 . 8 5 0 , 0 0}$ & $\mathbf{8 2 , 7 3}$ & $\mathbf{1 . 5 1 4 , 0 0}$ \\
\hline Pastaza & 0,00 & 0,00 & $\mathbf{0 , 0 0}$ & 0,00 \\
\hline Pichincha & $1.511,94$ & 828,10 & $\mathbf{5 4 , 7 7}$ & 73,42 \\
\hline Santa Elena & 401,29 & 85,12 & $\mathbf{2 1 , 2 1}$ & 4,76 \\
\hline Santo Domingo Tsáchilas & $1.618,34$ & $1.455,16$ & $\mathbf{8 9 , 9 2}$ & 230,35 \\
\hline *Sucumbíos & $\mathbf{1 3 . 6 6 6 , 0 0}$ & $\mathbf{1 1 . 9 9 0 , 0 0}$ & $\mathbf{8 7 , 7 4}$ & $\mathbf{1 . 5 3 6 , 0 0}$ \\
\hline Tungurahua & 0,00 & 0,00 & $\mathbf{0 , 0 0}$ & 0,00 \\
\hline *Zamora Chinchipe & $6.920,00$ & $4.200,00$ & $\mathbf{6 0 , 6 9}$ & 636,00 \\
\hline
\end{tabular}

* Los datos reflejados son hasta el año 2012

Fuente: (Sistema Nacional de Informacion).

En la tabla $\mathrm{N}^{\circ}$ 5, se puede observar que Manabí, Orellana y Sucumbíos poseen hasta la fecha de la data la mayor cantidad de hectáreas disponibles para el cultivo de café, destaca particularmente la provincia de Manabí que siendo la provincia con mayor cantidad de hectáreas disponibles tiene solo un $61,89 \%$ de sus hectáreas productivas, a diferencia de Sucumbíos y Orellana que tienen más del $80 \%$ de sus hectáreas productivas.

Imagen $\mathbf{N}^{\circ}$ 2: Producción nacional del café desde el año 2000 al 2014

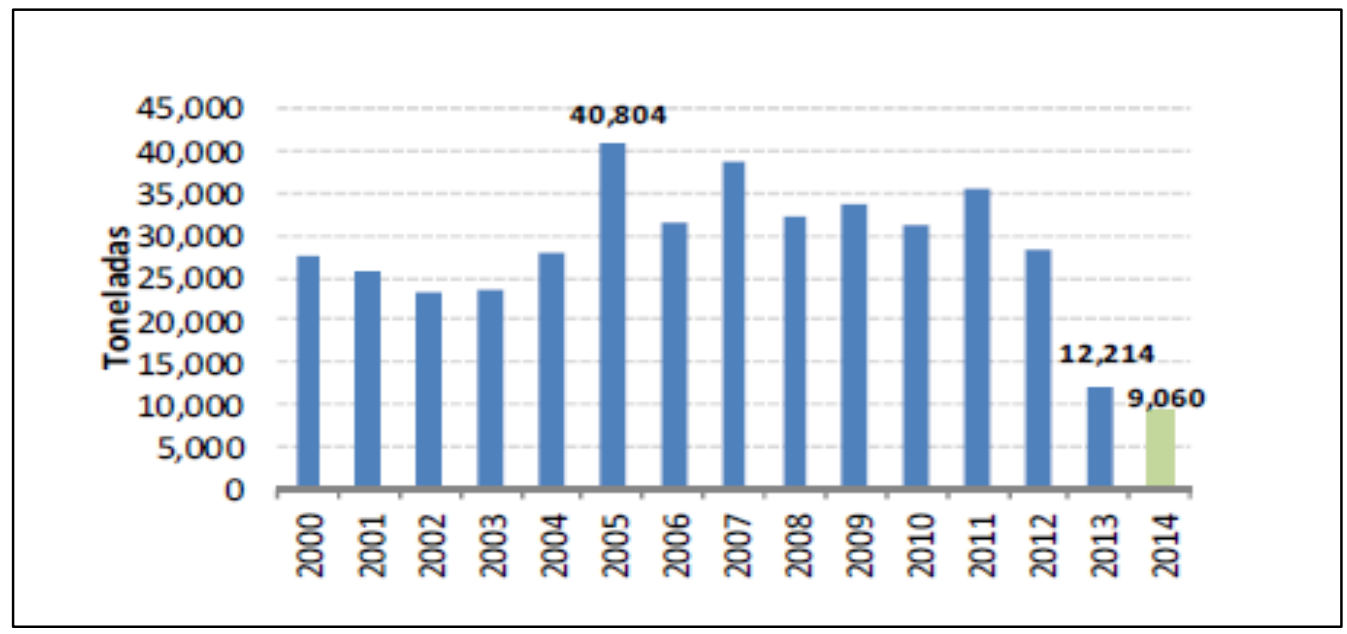

Fuente: (MAGAP, 2014, pag. 4) 


\section{La realidad Ecuatoriana en la producción de café}

Vol. 2, núm. 2., (2018)

Stefania Venegas Sánchez; Diego Orellana Bueno; Pablo Pérez Jara

La producción nacional del año 2014*, presentó similar comportamiento a la producción internacional, disminuyendo en $25.82 \%$ con respecto al año 2013. Debido a la reducción de la superficie cosechada. Esta variación negativa superó a la tasa promedio de crecimiento registrada en el periodo 2000-2013 (-2.58\%) (MAGAP, 2014, pag. 3)

Imagen $\mathbf{N}^{\circ}$ 3: Rendimiento Café 2015

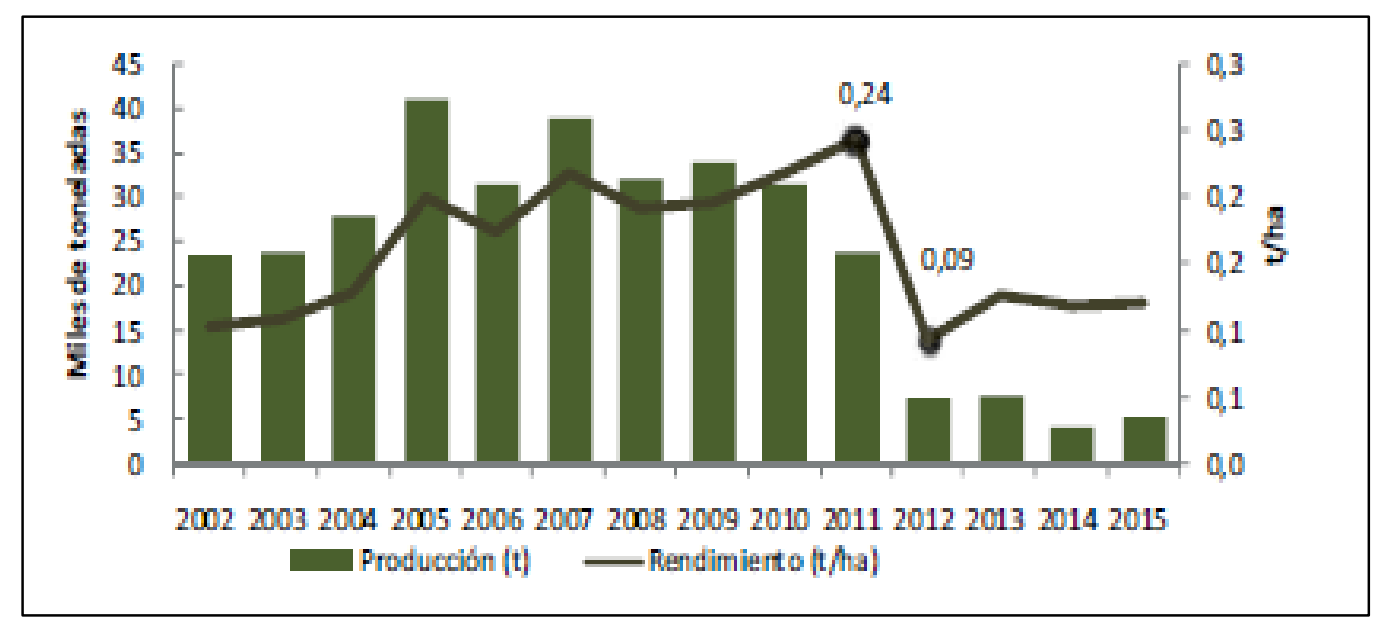

Fuente: (Monteros, 2016, pag. 1) 


\section{La realidad Ecuatoriana en la producción de café}

Vol. 2, núm. 2., (2018)

Stefania Venegas Sánchez; Diego Orellana Bueno; Pablo Pérez Jara

\section{Imagen $\mathbf{N}^{\circ}$ 4: Produccion Café en Ecuador por provincias.}

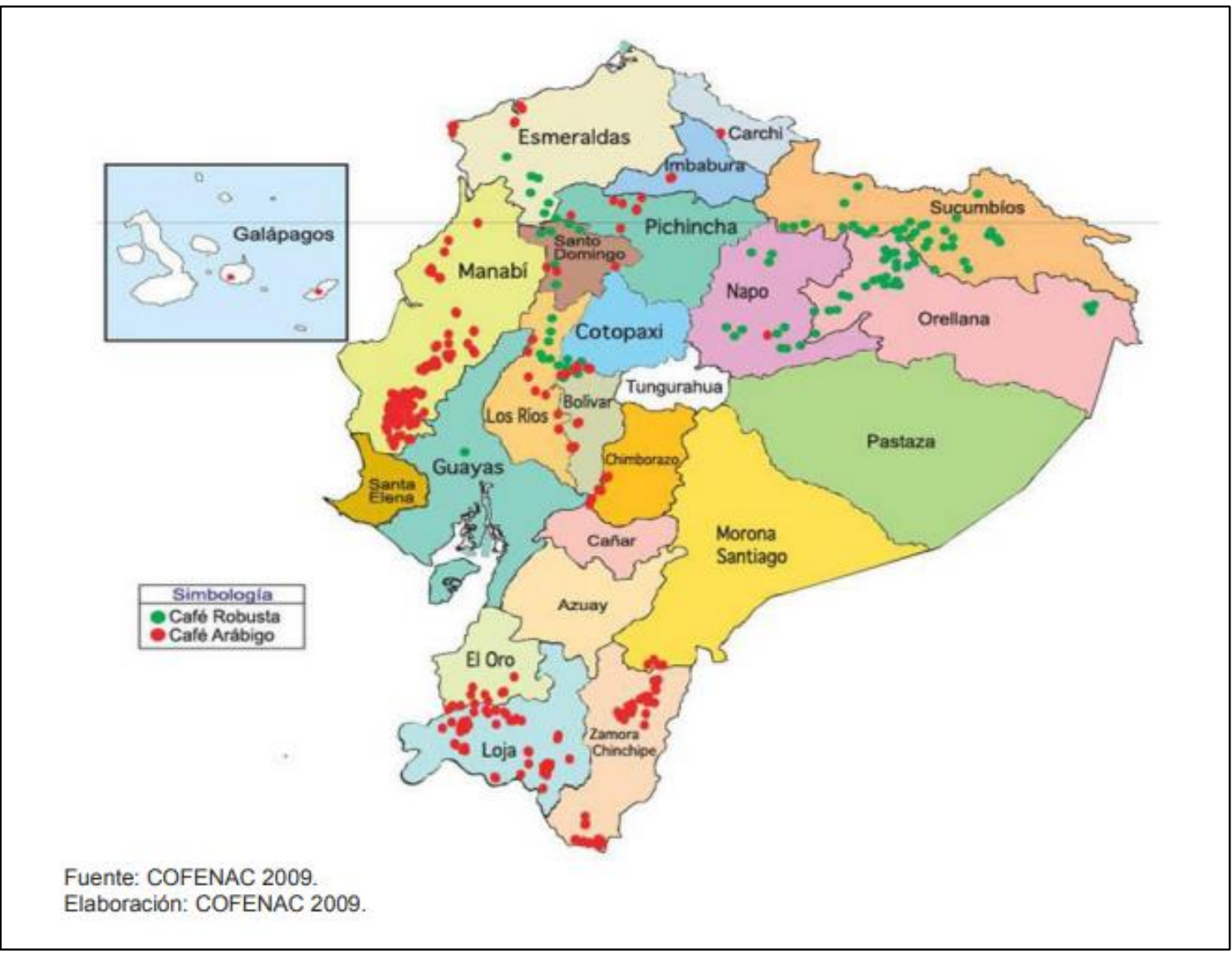

Fuente: (Pozo, 2014, pag. 34)

La baja productividad que caracteriza a los sistemas productivos de café en Ecuador se debe principalmente a la falta de conocimiento del germoplasma cultivado, a una mezcla de variedades o cultivares en las fincas productoras y a una incipiente tecnificación, que generalmente no considera una oportuna fertilización, aplicación del riego, prevención y control de enfermedades, desarrollo de podas sanitarias y de formación, renovación de cafetales que en la actualidad presentan una edad promedio de 25 años, etc. (COFENAC, 2011). A lo que hay que sumarle la gran crisis mundial del café de los años 1999 al 2002, que provoco el abandono del campo de muchos caficultores en busca de mejores condiciones de vida (Alarcó, 2011, pag. 15) 


\section{La realidad Ecuatoriana en la producción de café}

Vol. 2, núm. 2., (2018)

Stefania Venegas Sánchez; Diego Orellana Bueno; Pablo Pérez Jara

Por otra parte, la limitada capacidad organizativa de los productores (sólo el 5\% pertenecen a asociaciones de productores de café) y la gran presencia de intermediarios locales, que en muchas ocasiones introducen cascara de café para obtener mayores volúmenes, provocan grandes problemas en la comercialización e imagen externa del producto (Alarcó, 2011, pag. 15).

Durante el periodo 2001-2013, las exportaciones de café arábigo disminuyeron paulatinamente, excepto los cambios bruscos experimentados en los años 2006 y 2009 donde se observa un despunte en las exportaciones, atribuido a los efectos del proceso de renovación de cafetales arábigos, impulsado por el Consejo Nacional Cafetalero (COFENAC) y al repunte de los precios en el mercado internacional. Las exportaciones de café Robusta muestran variaciones significativas en los años 2005, 2009 y 2011, comportamiento inusual, originado, probablemente, por las crecientes importaciones de café Robusta provenientes de Vietnam (Vaca, Suarez, \& Velázquez, 2016, pag. 5).

En cuanto a la disminución tan vertiginosa de la producción de café Pozo (2014) tambien hace el siguiente comentario: La disminución de la producción de café está asociada según COFENAC a la falta de acceso a crédito, la falta de infraestructura para acceder a las plantaciones de café, infraestructura para riego, condicione climáticas. Otra razón importante de la disminución de la producción se ascia al desincentivo causado por el aumento de las importaciones de café consecuencia del régimen especial aduanero. A pesar de que el rendimiento del café ecuatoriano ha aumentado la producción ha disminuido. La producción de café se concentra en su mayoría en la Provincia de Manabí, seguido de la Provincia de Loja. (pág. 63). 


\section{La realidad Ecuatoriana en la producción de café}

Vol. 2, núm. 2., (2018)

Stefania Venegas Sánchez; Diego Orellana Bueno; Pablo Pérez Jara

Por otra parte, las exportaciones de café industrializado (Soluble y liofilizado) revelan un crecimiento sostenido durante el mismo período, además, se evidencia una tendencia hacia la baja de las exportaciones de café en grano y el despunte vertiginoso de las exportaciones de café industrializado en el período 2012-2014. En criterio del autor, las importaciones de café robusta sirven para cubrir la creciente demanda del sector industrial, toda vez que la producción nacional es deficitaria (Vaca, Suarez, \& Velázquez, 2016, pag. 5).

A partir del año 2013, la productividad del cultivo ha presentado signos de recuperación gracias a las políticas ejecutadas en su beneficio y de los agricultores. Es así, que para el año 2015 la producción de café se ubicó en 5 mil toneladas, con un rendimiento de 0.12 toneladas por hectárea (grano oro) (Monteros, 2016, pag. 1)

\section{Conclusiones.}

1. Los principales problemas de la caficultura ecuatoriana son la baja productividad de sus plantaciones, la deficiente calidad del grano y la falta del beneficio del grano por la vía húmeda (Vaca, Suarez, \& Velázquez, 2016, pag. 8).

2. La implementación de un Sistema Nacional de Innovación Tecnológica Cafetalera contribuirá al fortalecimiento del Plan Nacional de Reactivación de la Caficultura y ayudará a impulsar la transformación de la Matriz Productiva, expuesta en el objetivo $\mathrm{N}^{\circ} 10$ del Plan Nacional para el Buen Vivir 2013 -2017 (Vaca, Suarez, \& Velázquez, 2016, pag. 8).

3. El rendimiento objetivo promedio nacional de café Arábigo para el año 2016 fue de 0.22 toneladas por hectárea (Monteros, 2016, pag. 8) 


\section{La realidad Ecuatoriana en la producción de café}

Vol. 2, núm. 2., (2018)

Stefania Venegas Sánchez; Diego Orellana Bueno; Pablo Pérez Jara

4. El rendimiento objetivo promedio nacional de café Robusta para el año 2016 fue de 0.48 toneladas por hectárea. La provincia del Guayas fue la zona productora de mayor rendimiento (1.87 t/ha); mientras que, Napo fue la de menor productividad (0.08 t/ha) (Monteros, 2016, pag. 8).

5. La informacion estadistica publicada por el Instituo Nacional de Estadistica y Censos (INEC), esta muy desagreda para el tema de la produccion de café, las tablas presentadas contienen mucha informacion, pero en lo que respecta a esta investigación no fue de gran utilidad, ya que no se encontro la informacion buscada.

6. La pagina del Sistema Nacional de Informacion (SNI solo presenta datos estadisticos hasta el año 2013.

7. No hay mucha informacion oficial de la produccion de café del año 2017 , la pagina oficial del Consejo Nacional Cafetero (COFENAC) no tiene ningun tipo de estadisticas que se puedan visualizar o descargar.

8. En la pagina de la Asociacion Nacional de Exportadores de Café (ANECAFE) como su funcion lo indica solo arroja datos de exportacion, pero no muestra estadisticas de produccion.

9. Se busco series estadisticas que tuvieran un perioros desde el año 1990 hasta el año 2017 para observar el comportamiento real de la produccion de café en Ecuador, pero al momento de esta investigación no se encontro, publicandose tablas separadas y haciendoles los analisis correspondientes. 


\section{La realidad Ecuatoriana en la producción de café}

Vol. 2, núm. 2., (2018)

Stefania Venegas Sánchez; Diego Orellana Bueno; Pablo Pérez Jara

10. Hay mucha informacion de produccion pero no es muy confiable, ya que en varios trabajos leidos presentan cifras de un mismo año totalmente diferentes, lo que en dado caso le resta seriedad a cualquier investigación por que tiende a confundir a otros investigadores, en ese sentido hay que hacer una profunda investigación y escoger las mas confiables que en este sentido son las cifras oficiales.

\section{Recomendaciones.}

1. Se deben actualizar todos los portales que suminsitren informacion agroindustrial del Ecuador, hacer una investigación con datos no actualizados la limita, ya que no se pueden dar las conclusiones y recomendaciones en base a un real contexto de la situacion del tema de estudio.

2. Se debe retomar el sendero del cultivo del café de los años 90, y mas cuando existen varias provincias con un potencial enorme de produccion, dentro de las investigaciones para la realizacion de este trabajo se ha podido observar que Ecuador es un exportador de café y se debe seguir estimulando esa capacidad exportadora pero la unica manera es aumentando los niveles de produccion.

3. Hay que seguir capacitando a los productores de café, de igual maera hay que apoyarlos con financiamiento, semillas y fertilizantes. La calidad del café es importante, todos los entes cafetaleros junto con el Estado Ecuatoriano deben emplear los procesos tecnicos que tengan a la mano para logar en conjunto el objetivo de producir el mejor grano de café.

4. El Gobierno debe seguir impulsando la recuperacion de la produccion cafetera en el Ecuador, este pais tiene tradicion cafetalera desde los siglos XVII y XVIII. 


\section{La realidad Ecuatoriana en la producción de café}

Vol. 2, núm. 2., (2018)

Stefania Venegas Sánchez; Diego Orellana Bueno; Pablo Pérez Jara

5. Es necesaria la renovación masiva y nuevas siembras de cafetales en las áreas con mayor aptitud agroecológica. La renovación y nuevas siembras de cafetales debe realizarse usando variedades mejoradas de café arábigo, adaptados a los distintos agroecosistemas cafetaleros, que tengan el aval de instituciones de investigación y desarrollo cafetalero (Pozo, 2014, pag. $65)$.

\section{Bibliografía.}

Alarcó, A. (2011). Modelo de gestión productiva para el cultivo de café (COFFEA ARABICA L.) en el sur de Ecuador.

Guambi, L., Talledo, D., \& Ávila, E. (2016). Calidad organoléptica del café (Coffea arabica L.) en las zonas centro y sur de la provincia de Manabí, Ecuador. Revista española de estudios agrosociales y pesqueros, 15-34.

MAGAP. (2014). Boletin Situacional Cafe. Coordinación General del Sistema de Información Nacional (CGSIN).

Monteros, A. (2016). Rendimientos de café grano seco en el Ecuador 2016. Quito: MAGAP. Obtenido de http://sinagap. goricultura. ec/pdf/estudios_agroeconomicos/rendimiento_cafe_grano_seco2016. pdf.

Ortega, J. (2003). Análisis sectorial del café. Apuntes de Economía, 1-79.

Pozo, M. (2014). Análisis de los factores que inciden en la producción de café en el Ecuador 2000-2011.

Santistevan, M., Julca, A., Borjas, R., \& Tuesta, O. (2014). Caracterización de fincas cafetaleras en la localidad de Jipijapa (Manabí, Ecuador). Ecología Aplicada, 187-192.

Sistema Nacional de Informacion. (s.f.). Encuesta de Superficie y Produccion Agropecuaria 2003-2014.

Vaca, L., Suarez, K., \& Velázquez, I. (2016). Diagnóstico y propuesta de un sistema de innovación tecnológica cafetalera en Ecuador. Revista Cubana de Ciencias Forestales, $120-129$. 